\title{
APPLICATION OF SVD METHOD \\ IN SOLVING INCORRECT GEODESIC PROBLEMS
}

\author{
Andrii Sohor ${ }^{1}$ \\ Markiian Sohor ${ }^{2}$
}

DOI: https://doi.org/10.30525/978-9934-26-049-0-36

Abstract. The most reliable method for calculating linear equations of the least squares principle, which can be used to solve incorrect geodetic problems, is based on matrix factorization, which is called a singular expansion. There are other methods that require less machine time and memory. But they are less effective in taking into account the errors of the source information, rounding errors and linear dependence.

The methodology of such research is that for any matrix $A$ and any two orthogonal matrices $U$ and $V$ there is a matrix $\Sigma$, which is determined from the ratio. The idea of a singular decomposition is that by choosing the right matrices $U$ and $V$, you can convert most elements of the matrix to zero and make it diagonal with non-negative elements.

The novelty and relevance of scientific solutions lies in the feasibility of using a singular decomposition of the matrix to obtain linear equations of the least squares method, which can be used to solve incorrect geodetic problems.

The purpose of scientific research is to obtain a stable solution of parametric equations of corrections to the results of measurements in incorrect geodetic problems.

Based on the performed research on the application of the singular decomposition method in solving incorrect geodetic problems, we can summarize the following. A singular expansion of a real matrix $\bar{A}$ is any factorization $\bar{A}=\bar{U} \bar{\Sigma} \bar{W}^{T}$ of a matrix with orthogonal columns $\bar{U}$, an orthogonal matrix $\bar{W}$ and a diagonal matrix $\bar{\Sigma}$, the elements of which are called singular numbers of the matrix $\bar{A}$, and the columns of matrices $\bar{U}$

\footnotetext{
${ }^{1}$ Ph.D., Associate Professor,

Lviv Polytechnic National University, Ukraine

${ }^{2}$ Graduate Student of the Department of Cartography and Geospatial Modeling,

Lviv Polytechnic National University, Ukraine

(C) Andrii Sohor, Markiian Sohor
} 
and $\bar{W}$ left and right singular vectors. If the matrix $\bar{A}$ has a full rank, then its solution will be unique and stable, which can be obtained by different methods. But the method of singular decomposition, in contrast to other methods, makes it possible to solve problems with incomplete rank. Research shows that the method of solving normal equations by sequential exclusion of unknowns (Gaussian method), which is quite common in geodesy, does not provide stable solutions for poorly conditioned or incorrect geodetic problems. Therefore, in the case of unstable systems of equations, it is proposed to use the method of singular matrix decomposition, which in computational mathematics is called $S V D$. The $S V D$ singular decomposition method makes it possible to obtain stable solutions of both stable and unstable problems by nature. This possibility to solve incorrect geodetic problems is associated with the application of some limit $\tau$, the choice of which can be made by the relative errors of the matrix of coefficients of parametric equations of corrections $\bar{A}$ and the vector of results of geodetic measurements $\bar{L}$. Moreover, the solution of the system of normal equations obtained by the $S V D$ method will have the shortest length.

Thus, applying the apparatus of the singular decomposition of the matrix of coefficients of parametric equations of corrections to the results of geodetic measurements, we obtained new formulas for estimating the accuracy of the least squares method in solving incorrect geodetic problems. The derived formulas have a compact form and make it possible to easily calculate the elements $\mu$ and $Q_{X}$ estimates of accuracy, almost ignoring the complex procedure of rotation of the matrix of coefficients of normal equations.

\section{Introduction}

The most reliable method for calculating the coefficients in the general least squares problem is based on matrix factorization, which is called a singular decomposition. There are other methods that require less machine time and memory. But they are less effective in taking into account the errors of the source information, rounding errors and linear dependence [1].

Singular Value Decomposition or SVD is a powerful computational tool for analyzing matrices and matrix problems that has applications in many areas. This algorithm is a typical representative of the most currently used 
algorithms for solving various matrix problems on so-called eigenvalues and can be used simultaneously in numerical methods of these problems [2].

The methodology of such research is that for any matrix $A$ and any two orthogonal matrices $U$ and $V$ there is a matrix $\Sigma$, which is determined from the ratio $\Sigma=U^{T} A V$. If the elements $u_{j}$ and $v_{j}$ are columns of matrices $U$ and $V$, then the individual components of the matrix $\Sigma$ will be equal $\sigma_{i j}=u_{i}^{T} A v_{j}$.

The idea of a singular decomposition is that by properly choosing the matrices $U$ and $V$, most elements $\sigma_{i j}$ can be converted to zero; moreover, you can even make the matrix $\Sigma$ diagonal with non-negative elements.

It is little known that the singular decomposition has a long history. The works of the scientist Golub and his colleagues Kohan, Bizinger and Reinsch were fundamental in the vast majority. In particular, the article by Golub and Reinsh was published in 1971 [2]. The authors of the known algorithms for matrix eigenvalues are scientists Francis, Ruttishauser and Wilkinson; these algorithms were described in their works in 1965 [5]. In their work, scientists Lawson, Hanson (1974) and Stewart (1973) consider the SVD method and a number of related problems [3; 4].

Thus, the novelty and relevance of scientific solutions lies in the feasibility of using a singular decomposition of the matrix to obtain linear equations of the least squares method, which can be used to solve incorrect geodetic problems.

\section{Least squares data alignment}

Suppose that given $m$ points

$$
\left(t_{i}, y_{i}\right), \quad i=1, \ldots, m .
$$

We consider the value of $t$ as an independent variable, and the value of $y$ as a dependent associated with the value of $t$ by some unknown functional dependence

$$
y_{i}=y\left(t_{i}\right)
$$

We also assume that the value of $y$ must be approximated by a linear combination of $n$ given basic functions $\varphi_{j}$

$$
y(t) \approx c_{1} \phi_{1}(t)+c_{2} \phi_{2}(t)+\ldots+c_{n} \phi_{n}(t) .
$$

The linear combination in the right part of the approximate equation is called a linear mathematical model. The problem is to choose $n$ coefficients 
$c_{l}, \ldots, c_{n}$ so that the model is more or less consistent with the measurement results. The model is linear because the coefficients are included in it linearly, although the basis functions can be nonlinear functions from the parameter $t$.

The most common linear model is a polynomial

$$
y(t) \approx c_{1}+c_{2} t+c_{3} t^{2}+\ldots+c_{n} t^{n-1} .
$$

It is obtained if taken $\phi_{j}(t)=t^{j-1}$, although in practice other basic polynomials may be more favorable.

Among other cases of the linear model is the trigonometric approximation

$$
y(t) \approx c_{1} \sin t+c_{2} \sin 2 t+\ldots+c_{n} \sin n t,
$$

where $\phi_{j}(t)=\sin j t$.

Consider also the exponential approximation with fixed exponents

$$
y(t) \approx c_{1} e^{\lambda_{1} t}+c_{2} e^{\lambda_{2} t}+\ldots+c_{n} e^{\lambda_{n} t},
$$

in which $\phi_{j}(t)=\exp \left(\lambda_{j} t\right)$ for given $\lambda_{j}$. If you need to determine the value $\lambda_{j}$, then our model will become nonlinear.

Consider the case when the number of $m$ given points is greater than or equal to the number of $n$ unknown coefficients. In this case, the problem of choosing the coefficients is overdetermined and, as a rule, it is not possible to build a model that would accurately interpolate our measurement results.

Of the many different criteria for determining the coefficients $c_{j}$, the least squares method is most often used. For any choice of coefficients $c_{j}$, the residual at the $i$-th given point will be equal

$$
r_{i}=\sum_{j=1}^{n} c_{j} \phi_{j}\left(t_{i}\right)-y_{i} .
$$

The least squares principle requires that the coefficients $c_{j}$ be chosen from the condition of the minimum value of the sum of the squares of the residuals, that is

$$
\sum_{i=1}^{m} r_{i}^{2} \rightarrow \min .
$$

If the model exactly satisfies the measurement results, then this minimum will be zero, so that the interpolation here is considered as a special case.

The least squares principle does not necessarily define a single set of coefficients. If the basis functions are linearly dependent at given points, 
which means the presence of coefficients $\gamma_{j}$, among which there are nonzero, such that

$$
\sum_{j=1}^{n} \gamma_{j} \phi_{j}\left(t_{i}\right)=0, \quad i=1, \ldots, m
$$

then any multiple of the coefficients $\gamma_{j}$ can be added to the coefficients $c_{j}$ without changing the sum of the squares of the residuals. An important task in aligning the results of observations by the method of least squares with arbitrary basis functions is to identify such dependence and ambiguity.

There are many different algorithms for calculating a set of coefficients that gives the minimum sum of squares. One of the possibilities is the application of mathematical analysis [1].

Let

$$
r=\left(\sum_{i=1}^{m}\left(\sum_{j=1}^{n} c_{j} \phi_{j}\left(t_{i}\right)-y_{i}\right)^{2}\right)^{1 / 2} .
$$

Our goal is to minimize the value of $r$ or to minimize the value of $r^{2}$. To do this, the condition must be met

where $k=1, \ldots, n$.

$$
\frac{\partial r^{2}}{\partial c_{k}}=0
$$

Taking partial derivatives and changing the order of summation, we obtain

$$
\sum_{j=1}^{n}\left(\sum_{i=1}^{m} \phi_{j}\left(t_{i}\right) \phi_{k}\left(t_{i}\right)\right) c_{j}=\sum_{i=1}^{m} y_{i} \phi_{k}\left(t_{i}\right) .
$$

It is a system of $n$ linear equations with $n$ unknowns $c_{j}$. It can be written in matrix form

where

$$
P c=q,
$$

$$
\begin{gathered}
p_{k j}=\sum_{i=1}^{m} \phi_{k}\left(t_{i}\right) \phi_{j}\left(t_{i}\right), \\
q_{k}=\sum_{i=1}^{m} \phi_{k}\left(t_{i}\right) y_{i} .
\end{gathered}
$$

For example, when approximating by the method of least squares using a straight line

$$
y(t) \approx c_{1}+c_{2} t
$$


we have

$$
\begin{gathered}
P=\left(\begin{array}{cc}
m & \sum t_{i} \\
\sum t_{i} & \sum t_{i}^{2}
\end{array}\right), \\
q=\left(\begin{array}{c}
\sum y_{i} \\
\sum t_{i} y_{i}
\end{array}\right) .
\end{gathered}
$$

The equation $P_{c}=q$ is called normal equations. Note that the matrix $P$ depends only on the basis functions, because the values of $y_{i}$ are not included in it.

However, there are fundamental claims against the use of normal equations. It turns out that the matrix $P$ often has a very large number of conditions, so no matter how the normal equations are solved, the errors in the input information and rounding errors introduced in the solution process increase excessively in the calculated coefficients.

As a last resort, when the basis functions $\phi_{j}(t)$ are linearly dependent, we can say that the matrix $P$ is degenerate and the number of conditionality can be considered as leading to infinity. Therefore, methods that avoid a large number of conditionalities associated with normal equations are at the same time methods that can detect a linear relationship among the basic functions.

As we noted earlier, the most reliable method for calculating the coefficients on the principle of least squares, which is based on matrix factorization, is the method of singular decomposition or SVD method $[1 ; 2]$.

\section{Singular value decomposition}

The SVD method begins with the compilation of a matrix known in statistical analysis as the plan matrix. It is a rectangular matrix with the number of $m$ rows and the number of $n$ columns, the elements of which are equal

$$
a_{i j}=\phi_{j}\left(t_{i}\right) .
$$

If the value of $y$ denotes the vector of dimension $m$ with elements $y_{i}$, and the value of $c$ denotes the vector of dimension $n$ with elements $c_{j}$, then the approximate equations

$$
\sum_{j=1}^{n} c_{j} \phi_{j}\left(t_{i}\right) \approx y_{i}, \quad i=1, \ldots, m
$$


can be rewritten as

$$
A c \approx y .
$$

The singular decomposition of the original matrix $A$ gives us three matrices $\Sigma, U$ and $V$. The matrix $\Sigma$ is a diagonal matrix with non-negative diagonal elements, which are called singular numbers of the matrix $A$. The matrices $U$ and $V$ are used to convert equations

$$
A c \approx y
$$

into an equivalent diagonal system

$$
\Sigma \bar{c} \approx \bar{y} .
$$

Let $\sigma_{j}(j=1, \ldots, n)$ be the diagonal elements of the matrix $\Sigma$. In principle, if all elements $\sigma_{j}$ are nonzero, then the transformed equations can be solved as follows

$$
\bar{c}_{j}=\frac{\bar{y}_{j}}{\sigma_{j}}, \quad j=1, \ldots, n .
$$

But in practice it is not always possible to obtain such solutions if the values $\sigma_{j}$ are small.

It turns out that all values $\sigma_{j}$ are not equal to zero if and only if the basis functions $\phi_{j}$ are linearly independent at given points. Therefore, the key to the correct use of the SVD method is the introduction of some limit $\tau$, which reflects the accuracy of the original data. Any value $\sigma_{j}$ that is greater than the limit $\tau$ is acceptable and the corresponding element $\bar{c}_{j}$ is calculated as $\bar{y}_{j} / \sigma_{j}$. Any value $\sigma_{j}$ that is less than the limit $\tau$ is considered to be quite small and the corresponding element $\bar{c}_{j}$ can be given an arbitrary value. This arbitrariness of values is associated with the ambiguity of the set of coefficients obtained by the method of least squares. Changes in the input data and rounding errors that are less than the limit of $\tau$ can lead to a completely different set of coefficients. Since it is preferably desirable that these coefficients be as small as possible, we specify $\bar{c}_{j}=0$ if $\sigma_{j} \leq \tau$.

The relation $\sigma_{\max } / \sigma_{\min }$ where $\sigma_{\max }$ is the largest nonzero singular number and a $\sigma_{\min }$ is the smallest nonzero singular number can be considered as the conditionality number of the matrix $A$.

Dropping numbers $\sigma_{j}$ that are less than the limit of $\tau$ reduces the number of conditionalities to a value $\sigma_{\max } / \tau$. Since the number of conditionality is a factor in increasing the error, the result will be a more reliable determination of the coefficients $c_{j}$. The price of such reliability is a possible increase in residuals. 
Finally, it can be noted that an important feature of the SVD method is the ability to detect dependence and ambiguity and find very small singular numbers.

This type of additional information for least squares problems is called singular analysis. It is used in the analysis of complex mathematical models $[2 ; 5]$.

\section{Singular numbers and vectors}

In elementary linear algebra, a set of vectors is said to be linearly independent if none of them can be represented as a linear combination of the others. In computational linear algebra, it is very important to know the quantitative estimation of such independence $[1 ; 2]$.

Since two vectors are dependent if they are parallel, it is reasonable to consider them very independent if they are perpendicular or orthogonal. Two vectors $u$ and $v$ are called orthogonal if their scalar product is zero, that is

$$
u^{T} v=0 \text {. }
$$

The vector $u$ has length 1 if

$$
u^{T} u=1 .
$$

A square matrix is called orthogonal if its columns are pairwise orthogonal vectors of length 1 . Thus, the matrix $U$ is orthogonal if

$$
U^{T} U=I \text {, }
$$

where $I$ is a unit matrix. Note that the orthogonal matrix is automatically nondegenerate because $U^{-1}=U^{T}$.

Multiplication by orthogonal matrices does not change either the length of the vector or the angle between the two vectors. Orthogonal matrices are also characterized by the fact that they do not increase errors.

For any matrix $A$ and any two orthogonal matrices $U$ and $V$, consider the matrix $\Sigma$, which is determined from the ratio

$$
\Sigma=U^{T} A V .
$$

If the elements $u_{j}$ and $v_{j}$ are columns of matrices $U$ and $V$, respectively, then the individual components of the matrix $\Sigma$ will be equal

$$
\sigma_{i j}=u_{i}^{T} A v_{j} \text {. }
$$

The idea of a singular decomposition is that by properly choosing the matrices $U$ and $V$, most elements $\sigma_{i j}$ can be converted to zero; moreover, you can even make the matrix $\Sigma$ diagonal with non-negative elements. 
Thus, the singular expansion of a real matrix $A$ dimension $m \times n$ is called any of its factorization

$$
A=U \Sigma V^{T},
$$

where $U$ is an orthogonal matrix with dimension $m \times m, V$ is an orthogonal matrix with dimension $n \times n$, and $\Sigma$ is a diagonal matrix with dimension $m \times n$. The quantities $\sigma_{i}$ are called singular numbers of the matrix $A$, and the columns of the matrices $U$ and $V$ are called left and right singular vectors [5].

\section{Solving systems of linear equations}

Let $A$ be a given matrix of dimension $m \times n$, moreover $m \geq n$, and $b$ be a given vector of dimension $m$. You need to find all vectors $x$ for which

$$
A x=b \text {. }
$$

Moreover, we include here the case when the matrix $A$ can be square and degenerate.

Theoretically, there are many different algorithms that solve this system of equations. But in computational practice, with its inaccurate measurement results, the SVD method is essentially the only known method with high reliability of solutions $[1 ; 2 ; 3]$.

Using the singular decomposition of the matrix $A$, the linear system $A x=b$ can be rewritten as

$$
U \Sigma V^{T} x=b
$$

where to get

$$
\Sigma z=d,
$$

where $z=V^{T} x, d=U^{T} b$. The system of equations $\Sigma z=d$ is diagonal, which greatly simplifies its solution. This system can be divided into three subsystems, depending on the values of dimensions $m, n$ and rank $k$, ie the number of non-zero singular numbers:

$$
\begin{gathered}
\sigma_{j} z_{j}=d_{j} \text { if } j \leq n \text { and } \sigma_{j} \neq 0, \\
0 \cdot z_{j}=d_{j} \text { if } j \leq n \text { and } \sigma_{j}=0, \\
0=d_{j} \text { if } j>n .
\end{gathered}
$$

The second subsystem is empty if $k=n$; the third subsystem is empty if $n=m$.

The equations are compatible and the solution exists if and only if $d_{j}=0$ and also $\sigma_{j}=0$ or $j>n$. If $k<n$, then an unknown parameter 
$z_{j}$ corresponding to the zero coefficient $\sigma_{j}$ can be assigned an arbitrary value, then a solution of the system of equations can also be obtained. After returning to the original coordinates due to the transformation $x=V z$, arbitrary components $z$ allow to parameterize the set of all possible solutions $x$.

Denote by $u_{j}$ and $v_{j}$ the columns of the matrices $U$ and $V$. Then the decomposition $A=U \Sigma V^{T}$ can be written as follows:

$$
A v_{j}=\sigma_{j} u_{j}, \quad j=1, \ldots, n \text {. }
$$

The kernel of the matrix $A$ is the set of vectors $x$ for which $A x=0$ , and the range of values of the matrix $A$ is the set of vectors $b$ for which the system $A x=b$ has a solution. If $\sigma_{j}=0$, then $A v_{j}=0$ and $v_{j}$ belongs to the kernel of the matrix $A$; if so $\sigma_{j} \neq 0$, then $u_{j}$ belongs to the range of values of the matrix $A$.

It follows that we can obtain a complete description of the kernel and the range of values as follows. Let $V_{0}$ be a system of columns $v_{j}$ for which $\sigma_{j}=0$, and let $V_{1}$ be a system of other columns $v_{j}$. Let $U_{1}$ be a system of columns $u_{j}$ for which $\sigma_{j} \neq 0$, and let $U_{0}$ be a system of other columns $u_{j}$, including those for which $j>n$. In the system $V_{0}$ there are $n-k$ columns, in the system $V_{1}$ there are $k$ columns and as many in the system $U_{1}$, finally, in the system $U_{0}$ there are $m-k$ columns. Therefore:

1. $V_{0}$ is the orthonormal basis for the nucleus of the matrix $A$.

2. $V_{1}$ is the orthonormal basis for orthogonal complement of the nucleus $A$.

3. $U_{1}$ is the orthonormal basis for the range of values of the matrix $A$.

4. $U_{0}$ is the orthonormal basis for orthogonal complement of the range of values of $A$.

\section{The linear problem of the least squares method}

Let us now consider the generalization of the previous problem, but we will already look for vectors $x$ of dimension $n$, for which $A x$ it will only be approximately equal to the vector $b$ under the condition of the minimum length of the residual. By residual length here we mean a vector of dimension $m$, that is

$$
r=A x-b .
$$

Therefore, the task is to choose a vector $x$ that will minimize the length of the residual $r$ (or rather, the square of the length of the residual) 


$$
\|r\|^{2}=\sum_{i=1}^{m} r_{i}^{2} .
$$

This problem in statistics is called the linear regression problem. If the matrix $A$ has a full rank, then the solution $x$ will be unique and stable. This solution can be obtained using different methods than the SVD method. But the SVD method, unlike other methods, makes it possible to solve problems with incomplete rank $[1 ; 2 ; 5]$.

Since orthogonal matrices remain normal, then

$$
\|r\|=\left\|U^{T}\left(A V V^{T} x-b\right)\right\|=\|\Sigma z-d\| .
$$

Therefore, the SVD method reduces the general least squares problem to a diagonal matrix problem. It is not difficult to note that the vector $z$, which minimizes $\|r\|$, can be expressed by the following relations:

$$
\begin{gathered}
z_{j}=\frac{d_{j}}{\sigma_{j}} \text { if } \sigma_{j} \neq 0 ; \\
z_{j} \text { arbitrary if } \sigma_{j}=0 .
\end{gathered}
$$

Thus, $k$ equations of diagonal shape are solved exactly. Other equations boil down to the fact that the residual vector will be nonzero, and its norm will be equal to $\|r\|^{2}=\sum d_{j}^{2}$, where the sum is taken for all $j$, for which $\sigma_{j}=0$ or $j>n$. Then the inverse transformation $x=V z$ allows us to solve our original problem.

If the problem has an incomplete rank, then its solution, which minimizes $\|r\|$, will be non-unique. In this situation, we get a single solution by choosing $\|x\|^{2} \rightarrow \min$. This solution can be achieved if accepted

$$
z_{j}=0 \text { when } \sigma_{j}=0 .
$$

In the case of full rank, the solution will be the only one.

Modified least squares problems are often considered in practice, in which some combination of $\|r\|$ and $\|x\|$, that is

$$
\|r\|^{2}+\lambda\|x\|^{2} \rightarrow \min ,
$$

here $\lambda$ is some coefficient of proportionality.

\section{Solving incorrect geodetic problems}

The linear equations of the least squares method obtained in the previous paragraph can be used to solve incorrect geodetic problems. To do this, 
we represent the linear regression equation in the form of known in the alignment of geodetic networks of parametric equations of corrections. We present them in matrix form

$$
A X+L=V,
$$

where $A$ is the matrix of coefficients of parametric equations of corrections, the elements of which are obtained as partial derivatives of some function for unknown parameters; $X$ is a vector of unknown quantities, called parameters; $L$ is the vector of measurement results; $V$ is the vector of corrections to the measurement results.

This system of equations is indeterminate, so it does not have a single solution. Of the many different criteria for determining the unknown parameters of $X$, as we noted earlier, we use the principle of least squares. We impose an additional condition on the system of equations $A X+L=V$, that is

$$
\Phi=V^{T} C_{n n}^{-1} V \rightarrow \min ,
$$

where $C_{n n}^{-1}$ is the inverse covariance matrix of errors of measurement results.

In order to operate with simpler expressions of obtaining unknowns and their assessment of accuracy, it is advisable to use the representation of nonequivalent results of measurements in equivalent form.

If we substitute the condition of the minimum value of the vector $V$ from the parametric equations of corrections, we obtain

$$
\Phi=(A X+L)^{T} C_{n n}^{-1}(A X+L) \rightarrow \min .
$$

Opening parentheses, we will have

$$
\Phi=X^{T} A^{T} C_{n n}^{-1} A X+2 X^{T} A^{T} C_{n n}^{-1} L+L^{T} C_{n n}^{-1} L \rightarrow \min .
$$

For a valid symmetric positive definite inverse covariance matrix $C_{n n}^{-1}$ we use the following theorem.

Theorem. For a valid symmetric positive definite matrix $C_{n n}^{-1}$, there is such a symmetric positive definite matrix $C_{n n}^{-1 / 2}$ that $\left(C_{n n}^{-1 / 2}\right)^{2}=C_{n n}^{-1}$. Moreover, $\quad\left(C_{n n}^{-1 / 2}\right)^{2}=Y \Lambda^{1 / 2} Y^{T} \cdot Y \Lambda^{1 / 2} Y^{T}=Y \Lambda Y^{T}=C_{n n}^{-1}, \quad$ where $\Lambda^{1 / 2} \Rightarrow \operatorname{diag}\left\{\lambda_{1}^{1 / 2}, \lambda_{2}^{1 / 2}, \ldots, \lambda_{m}^{1 / 2}\right\}$ is the diagonal matrix of the eigenvalues of the matrix $C_{n n}^{-1 / 2}$, and the columns of the matrix $Y$ are the eigenvectors of the matrix $C_{n n}^{-1 / 2}$.

Using the definition of this theorem, the minimum condition can be represented as follows: 


$$
\Phi=X^{T} A^{T} C_{n n}^{-1 / 2} C_{n n}^{-1 / 2} A X+2 X^{T} A^{T} C_{n n}^{-1 / 2} C_{n n}^{-1 / 2} L+L^{T} C_{n n}^{-1 / 2} C_{n n}^{-1 / 2} L \rightarrow \min .
$$

If you enter the following notation

$$
\bar{A}=C_{n n}^{-1 / 2} A, \bar{L}=C_{n n}^{-1 / 2} L, \bar{V}=C_{n n}^{-1 / 2} V,
$$

then the condition of the minimum will finally take the form

$$
\Phi=X^{T} \bar{A}^{T} \bar{A} X+2 X^{T} \bar{A}^{T} \bar{L}+\bar{L}^{T} \bar{L}=\bar{V}^{T} \bar{V} \rightarrow \min .
$$

As is known from mathematical analysis, a necessary condition for the minimum of the function $\Phi$ is the zero equality of its partial derivatives, that is $\partial \Phi / \partial X=0$.

As a result, already for the case of equilibrium measurements $\bar{L}=C_{n n}^{-1 / 2} L$ we obtain a system of normal equations

$$
\bar{A}^{T} \bar{A} \cdot X+\bar{A}^{T} \bar{L}=0
$$

Unknown parameters are obtained here as

$$
X=-\left(\bar{A}^{T} \bar{A}\right)^{-1} \cdot \bar{A}^{T} \bar{L} .
$$

Research shows that the method of solving normal equations by sequential exclusion of unknowns (Gaussian method), which is quite common in geodesy, does not provide stable solutions for poorly conditioned or incorrect geodetic problems. Therefore, in the case of unstable systems of equations, it is desirable to use a method that would guarantee a stable solution. One of such methods can be a singular matrix decomposition, which in computational mathematics is called SVD $[1 ; 2]$.

Let us perform a singular expansion of the matrix $\bar{A}$. A singular decomposition of the real matrix $\bar{A}$ of dimension $m \times n$ is any factorization of the form

$$
\bar{A}=\bar{U} \bar{\Sigma} \bar{W}^{T}
$$

where $\bar{U}$ is a matrix with orthogonal columns of dimension $m \times n$, $\bar{W}$ is an orthogonal matrix of dimension $n \times n, \bar{\Sigma}$ is a diagonal matrix of dimension $n \times n$ in which $\bar{\sigma}_{i j}=0$ at $i \neq j$ and $\bar{\sigma}_{i j}=\bar{\sigma}_{j} \geq 0$. The quantities $\bar{\sigma}_{j}$ are called singular numbers of the matrix $\bar{A}$, and the columns of the matrices $\bar{U}$ and $\bar{W}$ are called left and right singular vectors. The following relations are valid for matrices $\bar{U}$ and $\bar{W}$

$$
\begin{aligned}
\bar{U}^{T} \bar{U} & =I, \bar{U} \bar{U}^{T} \neq I \\
\bar{W}^{T} \bar{W} & =I, \bar{W} \bar{W}^{T}=I,
\end{aligned}
$$

where $I$ is a unit matrix. 
Let us now substitute the value of the singular decomposition of the matrix $\bar{A}$ into a system of normal equations and obtain it

$$
\left(\bar{U} \bar{\Sigma} \bar{W}^{T}\right)^{T}\left(\bar{U} \bar{\Sigma} \bar{W}^{T}\right) \cdot X+\left(\bar{U} \bar{\Sigma} \bar{W}^{T}\right)^{T} \bar{L}=0
$$

After the actions of transposing matrices, we obtain

$$
\bar{W} \bar{\Sigma} \bar{U}^{T} \bar{U} \bar{\Sigma} \bar{W}^{T} \cdot X+\bar{W} \bar{\Sigma} \bar{U}^{T} \cdot \bar{L}=0
$$

After the reductions, the new system of normal equations will take the form

$$
\bar{W} \bar{\Sigma} \bar{\Sigma} \bar{W}^{T} \cdot X+\bar{W} \bar{\Sigma} \bar{U}^{T} \cdot \bar{L}=0 .
$$

Then the solution of such a system can be written as

$$
X=-\left(\bar{W} \bar{\Sigma} \bar{\Sigma} \bar{W}^{T}\right)^{-1} \bar{W} \bar{\Sigma} \bar{U}^{T} \cdot \bar{L} .
$$

Given the orthogonality of the matrix $\bar{W}$, and making the appropriate reductions, we finally obtain a solution of the system of normal equations

$$
X=-\bar{W} \bar{\Sigma}^{-1} \bar{U}^{T} \cdot \bar{L},
$$

where the matrix $\bar{\Sigma}^{-1}$ is a diagonal matrix whose members are equal $1 / \bar{\sigma}_{j}$.

Therefore, this solution makes it possible to obtain unknown parameters $X$ using a singular decomposition of the matrix $\bar{A}$.

We reduce it to an equivalent diagonal system

$$
\bar{\Sigma} \cdot Z=\bar{D}
$$

where

$$
\begin{aligned}
& Z=\bar{W}^{T} X, \\
& \bar{D}=-\bar{U}^{T} \bar{L} .
\end{aligned}
$$

If all values $\bar{\sigma}_{j}$ are nonzero, then the equivalent diagonal system can be solved by assuming

$$
z_{j}=\frac{\bar{d}_{j}}{\bar{\sigma}_{j}}, \quad j=1, \ldots, n,
$$

where $z_{j}$ are the elements of the vector $Z, \bar{d}_{j}$ are the elements of the vector $\bar{D}$.

However, in practice it is not always possible to obtain a correct solution to such a problem if some values $\bar{\sigma}_{j}$ are small.

According to the statements described in the previous paragraphs, we can say that all values of $\bar{\sigma}_{j}$ will not be equal to zero in the case when the columns of the elements $\bar{a}_{i j}$ of the matrix $\bar{A}$ are linearly independent.

As we noted earlier, to obtain a correct solution, you need to enter some limit $\tau$, which reflects the accuracy of the original data used in geodetic 
problems. Changes in the original data and rounding errors that are less than the limit of $\tau$ can lead to a completely different set of coefficients. Given that these coefficients are as small as possible, we can write

$$
z_{j}=0 \text { if } \bar{\sigma}_{j} \leq \tau \text {. }
$$

The choice of $z_{j}=0$ has a special meaning. It results in a solution that has the shortest length of all possible solutions.

The relation $\bar{\sigma}_{\max } / \bar{\sigma}_{\min }$ is called the number of conditionality of the matrix $\bar{A}$, that is

$$
\operatorname{cond}(\bar{A})=\frac{\bar{\sigma}_{\max }}{\bar{\sigma}_{\min }},
$$

where $\bar{\sigma}_{\max }$ is the largest nonzero singular number, and $\bar{\sigma}_{\min }$ is the smallest nonzero singular number.

Therefore, rejecting the numbers $\bar{\sigma}_{j}$ that will be less than the limit $\tau$, we obtain a decrease in the number of conditionality to the value $\bar{\sigma}_{\text {max }} / \tau$, that is

$$
\operatorname{cond}(\bar{A})=\frac{\bar{\sigma}_{\max }}{\tau} .
$$

Studies have shown that discarding some small values $\bar{\sigma}_{\dot{j}}$ is appropriate when the number of conditionality of the matrix cond $(\stackrel{\dot{A}}{)})$ exceeds the inverse relative errors of the matrix $\bar{A}$ and the vector of free terms $\bar{L}$, which is the vector of the results of measurements of geodetic quantities.

Then, we can obtain a stable solution of our problem if the following condition is met

$$
\operatorname{cond}(\bar{A}) \leq d,
$$

where $d$ is the denominator of the relative errors of the matrix $\bar{A}$ and the vector of geodetic measurements $\bar{L}$.

If we substitute the value of $\operatorname{cond}(\bar{A})=\bar{\sigma}_{\max } / \tau$ into the inequality $\operatorname{cond}(\bar{A}) \leq d$, we obtain

$$
\frac{\bar{\sigma}_{\max }}{\tau} \leq d .
$$

Then the boundary $\tau$ can be determined from the condition

$$
\tau>\frac{\bar{\sigma}_{\max }}{d} .
$$

Thus, we have shown the problem of solving incorrect geodetic problems in the form of a system of normal equations, which makes it possible, given the original covariance matrix $C_{n n}$, to obtain a fairly simple expression for finding unknown quantities $X$, using a singular matrix decomposition 
of parametric equations $\bar{A}$. The singular decomposition method makes it possible to obtain stable solutions of both stable and unstable problems by nature. This ability to solve incorrect geodetic problems is associated with the use of some limit $\tau$, which allows not to include in the calculation process very small singular numbers $\bar{\sigma}_{j}$ and thus improve the conditionality of the system of normal equations. The choice of the limit $\tau$ can be made by the relative errors of the matrix of coefficients of parametric equations of corrections $\bar{A}$ and the vector of results of geodetic measurements $\bar{L}$. Moreover, the solution of the system of normal equations obtained by the SVD method will have the shortest length.

\section{Estimated accuracy}

We derive formulas for estimating accuracy using the singular decomposition method.

In general, the root mean square error of any quantity is determined from the formula

$$
m_{X}=\mu \sqrt{Q_{X}},
$$

where $\mu$ is the error per unit weight, $Q_{X}=P_{X}^{-1}$ is the inverse weight of the vector of the estimated value.

Thus, the task of estimating accuracy is decomposed into two tasks: 1) determining the error of a unit of weight; 2) determining the weight of the vector of the estimated value.

To determine the error of a unit of weight, you can use the formula from the theory of errors

$$
\mu=\sqrt{\frac{\bar{V}^{T} \bar{V}}{m-n}},
$$

where $m-n=k$ is the number of excessively measured values, and the product of matrices $\bar{V}^{T} \bar{V}$, according to the formulas of the previous paragraph, is equal to $V^{T} C_{n n}^{-1} V$. Using the values of the function $\Phi=\bar{V}^{T} \bar{V}$ given above, and performing some simplifications, we obtain

$$
\bar{V}^{T} \bar{V}=X^{T}\left(\bar{A}^{T} \bar{A} X+\bar{A}^{T} \bar{L}\right)+\bar{L}^{T} \bar{A} X+\bar{L}^{T} \bar{L} .
$$

But the expression that is in parentheses is zero because it represents a system of normal equations. Therefore, the value $\bar{V}^{T} \bar{V}$ will take the form

$$
\bar{V}^{T} V=\bar{L}^{T} \bar{A} X+\bar{L}^{T} \bar{L} .
$$


Then, using the singular decomposition of the matrix $\bar{A}$, this formula can be represented as

$$
\bar{V}^{T} V=\bar{L}^{T} \bar{U} \bar{\Sigma} \bar{W}^{T} X+\bar{L}^{T} \bar{L} .
$$

If we replace the vector $X$ with its value obtained from the solution of the system of normal equations, then we have

$$
\bar{V}^{T} V=-\bar{L}^{T} \bar{U} \bar{\Sigma} \bar{W}^{T} \bar{W} \bar{\Sigma}^{-1} \bar{U}^{T} \bar{L}+\bar{L}^{T} \bar{L} .
$$

Using the properties of the transposed, orthogonal and inverse matrices, the value $\bar{V}^{T} \bar{V}$ is written

$$
\bar{V}^{T} V=-\bar{L}^{T} \bar{U} \bar{U}^{T} \bar{L}+\bar{L}^{T} \bar{L} .
$$

Then the root mean square error of the unit of weight will finally take the form

$$
\mu=\sqrt{\frac{\bar{L}^{T} \bar{L}-\bar{L}^{T} \bar{U} \bar{U}^{T} \bar{L}}{m-n}} .
$$

For the inverse weight $Q_{X}$ in the theory of errors take the value

$$
Q_{X}=\left(A^{T} C_{n n}^{-1} A\right)^{-1} .
$$

Using the theorem of symmetric positive definite matrix $\left(C_{n n}^{-1 / 2}\right)^{2}=C_{n n}^{-1}$ formulated in the previous paragraph, the inverse weight $Q_{X}$ can be written

$$
Q_{X}=\left(\bar{A}^{T} \bar{A}\right)^{-1} .
$$

After performing a singular decomposition of the matrix $\bar{A}$, the inverse weight $Q_{X}$ will take the form

$$
Q_{X}=\left[\left(\bar{U} \bar{\Sigma} \bar{W}^{T}\right)^{T} \bar{U} \bar{\Sigma} \bar{W}^{T}\right]^{-1} .
$$

After transposing and simplifying the expression, the value of $Q_{X}$ will be written

$$
Q_{X}=\left[\bar{W} \bar{\Sigma} \bar{\Sigma} W^{T}\right]^{-1} .
$$

Using the properties of the inverse matrix of the product and the orthogonal matrix, we finally obtain the inverse weight of the vector of the estimated value

$$
Q_{X}=\bar{W} \bar{\Sigma}^{-1} \bar{\Sigma}^{-1} W^{T} .
$$

Thus, applying the apparatus of the singular decomposition of the matrix of coefficients of parametric equations of corrections to the results of geodetic measurements, we obtained new formulas for estimating the 
accuracy of the least squares method in solving incorrect geodetic problems. The derived formulas have a compact form and make it possible to easily calculate the elements $\mu$ and $Q_{X}$ of the accuracy estimate, almost ignoring the complex procedure of rotation of the matrix of coefficients of normal equations.

\section{Conclusions}

Based on the research on the application of the SVD method in solving incorrect geodetic problems, we can summarize the following:

1. A singular decomposition of a real matrix $\bar{A}$ is any factorization of $\bar{A}=\bar{U} \bar{\Sigma} \bar{W}^{T}$ into a matrix with orthogonal columns $\bar{U}$, an orthogonal matrix $\bar{W}$ and a diagonal matrix $\bar{\Sigma}$, the elements of which $\bar{\sigma}_{j}$ are called singular numbers of matrix $\bar{A}$, and columns of matrices $\bar{U}$ and $\bar{W}$ are left and right singular vectors.

2. If matrix $\bar{A}$ has a full rank, then its solution will be unique and stable, which can be obtained by different methods. But the method of singular decomposition, in contrast to other methods, makes it possible to solve problems with incomplete rank.

3. Research shows that the method of solving normal equations by sequential exclusion of unknowns (Gaussian method), which is quite common in geodesy, does not provide stable solutions for poorly conditioned or incorrect geodetic problems.

4. Therefore, in the case of unstable systems of equations, it is proposed to use the method of singular matrix decomposition, which in computational mathematics is called SVD.

5. The method of singular matrix decomposition or SVD makes it possible to obtain stable solutions of both stable and unstable problems by nature. This possibility to solve incorrect geodetic problems is associated with the application of some limit $\tau$, the choice of which can be made by the relative errors of the matrix of coefficients of parametric equations of corrections $\bar{A}$ and the vector of geodetic measurements $\bar{L}$. Moreover, the solution of the system of normal equations obtained by the SVD method will have the shortest length.

6. We have obtained new formulas for estimating the accuracy of the SVD method in solving incorrect geodetic problems. The derived formulas have a compact form and make it possible to easily calculate the elements 
$\mu$ and $Q_{X}$ of the accuracy estimate, almost ignoring the complex procedure of rotation of the matrix of coefficients of normal equations.

\section{References:}

1.Forsythe G.E., Malcolm M.A., Moler C.B. (1977) Computer Methods for Mathematical Computations. Englewood Cliffs. N.J. Prentice-Hall.

2. Golub G.H., Reinsch C. (1971) Singular Value Decomposition and Least Squares Solution, in J.H. Wilkinson and C. Reinsch (eds.), Handbook for Automatic Computation, vol. II: Linear Algebra. Heidelberg. Springer.

3.Lawson C.L., Hanson R.J. (1974) Solving Least Squares Problems. Englewood Cliffs. N.J. Prentice-Hall.

4. Stewart G.W. (1973) Introduction to Matrix Computation. New York: Academic Press. Press.

5. Wilkinson J.H. (1965) The Algebraic Eigenvalue Problem. Oxford: Clarendon 